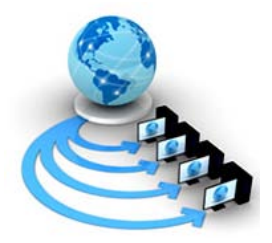

Volume 9, No. 2, March-April 2018

RESEARCH PAPER

Available Online at www.ijarcs.info

\title{
LEARN ON MAPREDUCE METHOD FOR JOB SCHEDULING BY USING BIGDATA
}

\author{
G. Suhasini \\ Research scholar \\ mewar university, Chittorgarh, \\ Rajasthan, India
}

\author{
Dr. P. Niranjan \\ Research supervisor \\ mewar university, Chittorgarh, \\ Rajasthan, India
}

\begin{abstract}
Many companies are increasingly using Map Reduce for inexperienced massive scale data processing together with personalized marketing , direct mail detection, and brilliant data mining obligations. Cloud computing offer an attractive desire for corporations to hire an appropriate length Hadoop cluster, use resources as a company, and pay simplest for property that has been implemented. One of the open questions in such environments is the amount of property that someone ought to rent from the service provider. Often, a patron goals particular well-known normal performance dreams and the software program desires to entire facts processing by means of the manner of way of a positive time cut-off date. However, currently, the mission of estimating required assets to satisfy software program, overall performance desires is most effective the clients' obligation. In these, we introduce a unique framework and approach to deal with this problem and to provide a modern beneficial resource sizing and provisioning service in Map Reduce environments. For a Map Reduce technique that desires to be finished in interior a positive time, the challenging profile is constructed from the technique past executions or by way of executing the utility on a smaller statistics set the use of an automatic profiling device. Map Reduce application is used to acquire data in line with the request. To approach massive facts proper scheduling is required to gain extra well-known overall performance. Scheduling is a way of assigning jobs to available assets in a way to decrease hunger and maximize resource usage. Performance of scheduling technique may be advanced with the useful resource of using lessen-off date constraints on jobs. The goal is to examine Map Reduce and notable scheduling algorithms that can be used to benefit better overall performance.
\end{abstract}

Keywords: Map Reduce, technique, environment, algorithm.

\section{INTRODUCTION}

A cloud scheduler plays a primary feature in shelling out assets for distinctive jobs executing in a cloud environment. Virtual machines are created and controlled on the fly in the cloud to create surroundings for method execution. Map Reduce is an easy and effective programming model which has been notably used for processing large-scale information first-rate applications on a cluster of physical machines. Now a day's many businesses, researchers, government businesses are strolling Map-Reduce applications on the public cloud. Running Map Reduce on the cloud has many benefits like on-call for set up the order of cluster, scalability[1]. Many Map Reduce Frameworks like Google Map Reduce, Dry and, are to be had however the open deliver Hadoop Map Reduce is typically used. But taking walks a Hadoop cluster on a private cluster isn't always similar to walking on a public cloud[2]. Public cloud allows having digital cluster in which resources can be provisioned or released as in step with the requirement of the software in mines. Executing Map Reduce packages on cloudlets in the customer to execute jobs of several requirements without taking any ache of making and retaining a cluster[3]. Scheduling performs a primary feature inside the ordinary widespread performance of Map-Reduce Applications. The default scheduler in Hadoop Map Reduce is FIFO Scheduler, Face book uses Fair Scheduler, and Yahoo makes use of Capacity Scheduler[4]. The above schedules are regular examples of schedules for Map Reduce software application are exquisite appropriate for bodily static clusters, that also can serve the cloud systems with dynamic beneficial resource manage, but the one's schedules do not take into account the competencies suffering from virtualization used in cloud environments[5]. Therefore, those are a need of dynamic scheduler that could time desk Map Reduce packages primarily based totally on the features of the software program application, Virtual Machines, and locality of input statistics to properly execute those programs in hybrid cloud surroundings.

\section{PREVIOUS STUDY}

This section gives an assessment of the literature on task scheduling and useful resource provisioning in cloud computing with respect to Map Reduce programming paradigm. Assuncao et al. [5] explored awesome inclinations in cloud computing and massive records analytics. They specified 4 elements of analytics which incorporates architectural manual and facts control, version improvement, customer interplay and visualization, and commercial enterprise models. They labeled analytics into descriptive, predictive and attitude. A descriptive model is used for modeling beyond behavior. Predictive is for forecasting primarily based mostly on the existing records and attitude is for selection making and assessing movements[6]. They also mentioned about the characteristics of massive statistics which includes variety, velocity, extent, veracity, and fee. Variety refers to records types. Velocity refers to data manufacturing and processing pace. Volume refers to the scale of information. Veracity refers to records reliability and believes. Value refers honestly really worth acquired from massive facts after analysis. Job scheduling performs critical feature in Map Reduce programming. Mashayekhy et al. [7] studied the concept of energy-conscious scheduling of Map Reduce jobs for large information applications. They proposed and carried out a framework to have strength-conscious pastime scheduling for big facts packages with service degree 
agreements (SLA). They moreover proposed heuristic algorithms referred to as electricity-conscious Map Reduce scheduling algorithms[8]. They deal with the task of the map and decrease obligations as a way to optimize the electricity ate up for Map Reduce programming[9]. They used one of kind algorithms for Map Reduce programming in Hadoop allotted environment for finding execution time and power consumption. They tested algorithms with special workloads and found the overall performance of the proposed algorithms in on foot map and reduce obligations with energy-focus[10].

\section{METHODOLOGY}

MapReduce is a framework for the parallel processing of huge information in a dependable, fault-tolerant way on big clusters. The data need to be clustered based absolutely totally on their priorities, facts dependence, final date timetable for processing and processing of statistics clusters. For example, if the processing of one fact dreams the output of some other records as entering then the ones can be mixed to shape a cluster. The MapReduce framework consists of a preserve close to Job Tracker and slave Task Tracker. The Job Tracker gives beneficial resource manipulate which incorporates timetable the responsibilities on the slave Task Trackers, display and re-execute the failed duties and beneficial resource intake/availability. The slave Task Trackers execute duties and supply challengepopularity records to the draw close periodically. There is a single component of failure, because of this if Job Tracker fails; all taking walks jobs are halted. In case of a single node failure, map obligations and incomplete lessen duties may be completed in place of the entire map obligations and reduce duties to accumulate the minimum execution time.

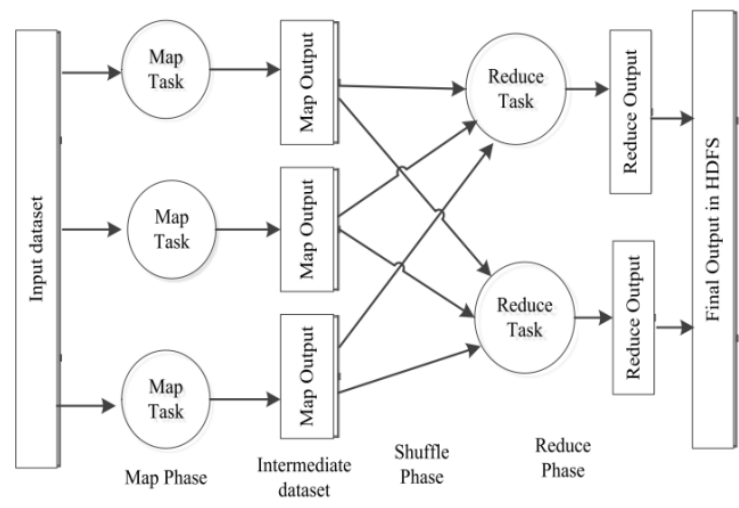

Fig.3.1. MapReduce execution model

The execution begins with a dataset given as entering. Once the dataset is given the Map phase begins of evolved. The Map section consists of more than one Map responsibilities that act on given information in parallel and bring intermediate output. The intermediate dataset is subjected to shuffling or sorting. Then the output of shuffling is given to reduce phase. In the lessen segment, the final output is generated and saved in Hadoop Distributed File System (HDFS). The version includes Map section version, Reduce section model and Shuffling phase model. In the ones 3 levels, the modeling considers unmarried jobs, a couple of jobs within the single wave and multiple waves. The reason of the model is to estimate assignment execution time and feature optimized procedure scheduling and useful aid provisioning. This model can lessen over-provisioning as it considers the above-said environment. It is anticipated to paintings first-class with jobs with prolonged remaining dates with appreciate to execution.

\section{HADOOP DISTRIBUTED FILESYSTEM}

Hadoop can use any of the allotted record devices including HFTP FS, Local FS, S3 FS, but the document device utilized by Hadoop is known as Hadoop Distributed File System (HDFS). The HDFS depends at the Google File System (GFS) and offers a dispensed record system to run an application in a fault-tolerant and reliable manner on large clusters (hundreds of computers) of small laptop machines. HDFS makes use of hold near/slave structure. Master is expressed with the aid of a single Name Node that shops the document machine metadata. One or extra slave Data Nodes shop the true facts. The Data Nodes observes and write operation with the HDFS. They moreover perform block introduction, replication, and deletion based totally on schooling certain with the beneficial useful resource of Name Node. A document is partitioned into several blocks and those blocks are stored in the set of Data Nodes. The Name Node unearths out the mapping of blocks to the Data Nodes. To correctly take the gain of parallel execution of massive facts applications using Map Reduce on the cloud, there can be a call for of designing a scheduler which gives an excessive regular usual overall performance without a compromise on manageability, fault tolerance.

\section{CONCLUSION}

To conquer the difficulty of Big Data storage and processing the open supply framework named Hadoop is advanced through Apache may be used. Hadoop gives a deliver to Big Data processing with its components like Map Reduce and HDFS. To device with the Big Data the default scheduler known as FIFO has been used. Different scheduling techniques to enhance the statistics locality, makespan, standard overall performance, equity and regular performance are noted. Scheduling may be made inexperienced via using the statistics of records locality of the intermediate data generated with the aid of the map duties. This records permits out to lessen the intermediate community site visitors within the direction of the reduced section and there via using way of rushing the execution of map lessen programs.

\section{REFERENCES}

[1] Sandholm T, Lai K - Dynamic proportional percentage scheduling in hadobpProceedings of the Fifteenth Workshop on Job Scheduling Strategies for Parallel Processing, Vol-6253, pp. One hundred ten-131, 2010, ISBN: 978- 3-642-16504-7.

[2] Chen Y, Ganapathi A, Griffith R, Katz RH - The case for evaluating MapReduce regular basic performance the usage of workload suites Proceedings of the 19th Annual IEEE/ACM International Symposium on Modeling, Analysis and Simulation of Computer and Telecommunication Systems, Washington, pp. 390-399, 2011, ISSN: 1526- 7539.

[3] Polo J, Castillo C, Carrera D, Becerra Y, Whalley I, Steiner M, Torres J, Ayguade E - Resource-Aware Adaptive 
Scheduling for MapReduce Cluster\$ Middleware, LNCS 7049, pp. 187-207, 2011, ISSN: 0302- 9743.

[4] Wolf J, Balmain A, Rajan D, Hildrum K, Khandekar R, Parekh S, Wu KL, Veronica R - On the optimization of schedules for MapReduce workloads inside the presence of shared scanls The VLDB Journal, Vol -21, pp. 589609, 2012, ISSN: 1066-8888.

[5] Tang Z, Zhou J, Li K, Li R - A MapReduce assignment scheduling set of guidelines for ultimate date constraints Cluster Comput, pp. 651-662,2012, ISSN: 1386-7857.

[6] Song G, Yu L, Meng Z, Lin X - A Game Theory-Based MapReduce Scheduling Algorittlimerging

Technologies for Information Systems, Computing, and Management, Vol-236, pp. 287-296, 2013, ISBN: 978-14614-7009-zero.
[7] Chen H, Shen Y, Chen Q, Guo M - HMHS: Hybrid Multistage Heuristic Scheduling Algorithm for Heterogeneous MapReduce SystemICA3PP, Part I, LNCS 8285, pp. 196-205, 2013, ISSN: 0302- 9743.

[8] Zhao Y, Chen L, Li Y, Liu P, Li X, Zhu C - RAS: A Task Scheduling Algorithm Based on Resource Attribute Selection in a Task Scheduling Framework IDCS, LNCS 8223, pp. 106-119, 2013, ISSN: 0302- 9743.

[9] Yuan Z, Wang J - Research of Scheduling Strategy Based on Fault Tolerance in Hadoop Platform\| GRMSE, Part II, CCIS 399, pp. 509-517, 2013, ISSN: 1865- 0929. [10] swathi baswaraju-Map Reduce based Analysis of Live Website Traffic integrated with improved Performance for Small files using Hadoop, IJSRD,volume 4,issue 1,pages1496-1498 